\title{
Thionine Dye Confined in Zeolite L: Synthesis Location and Optical Properties
}

\author{
Lara Gigli, ${ }^{\dagger}$ Rossella Arletti, ${ }^{, \dagger}, \ddagger$ Jenny G. Vitillo, ${ }^{\ddagger, \S}$ Gabriele Alberto, ${ }^{\ddagger}, \|$ Gianmario Martra, ${ }^{\ddagger}, \|$ \\ André Devaux ${ }^{\perp}$ and Giovanna Vezzalini ${ }^{\#}$ \\ ${ }^{\dagger}$ Dipartimento di Scienze della Terra, Università degli Studi di Torino, Via Valperga Caluso 35, 10125 Torino, Italy \\ ${ }^{*}$ Nanostructure Interfaces and Surfaces, Centre of Excellence, Via Quarello 15A, 10135 Torino, Italy \\ ${ }^{\S}$ Dipartimento di Scienze ad Alta Tecnologia, Università degli Studi dell'Insubria, Via Valleggio, 11, 22100 Como, Italy \\ "Dipartimento di Chimica, Università degli Studi di Torino, Via Pietro Giuria 7, 10125 Torino, Italy \\ ${ }^{\perp}$ Department of Chemistry, University of Fribourg, Chemin du Musée 9, CH-1700 Fribourg, Switzerland \\ \#Dipartimento di Scienze Chimiche e Geologiche, Università degli Studi di Modena, Via Giuseppe Campi 103, 41125 Modena, Italy
}

\begin{abstract}
The process of light conversion is at present deeply investigated to develop more efficient devices for artificial photosynthesis, water splitting, photovoltaic applications, and targeting therapeutic agents. This process is induced by nanostructured functional materials, such as zeolites light sensitized by dye molecules adsorbed into their pores. Here a detailed study of the organization of the cationic dye thionine $(\mathrm{Th})$ inserted into the linear $12 \mathrm{MR}$ channels of zeolite L is carried out by XRPD, FT-IR spectroscopy-for the determination of the host-guest interactions-and UV-vis absorption and photoluminescence spectroscopies for the investigation of the electronic states of the guest. Two composites with different Th loading (labeled ZL/0.15Th and ZL/0.27Th) were obtained through ion exchange. TGA spectra and IRATR spectroscopy clearly indicated penetration of Th molecules into the ZL channels. Rietveld structural refinement showed that Th molecules in both

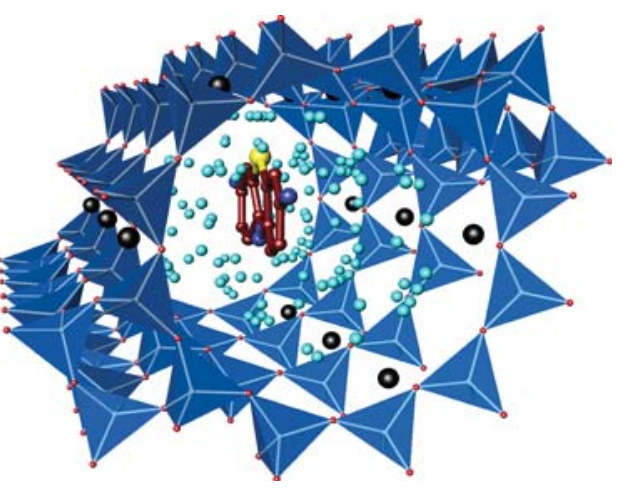
composites are aligned parallel to the 12MR channel axis and located on the mirror planes parallel to the $c$-axis. Water molecules interact strongly with the dye and form a kind of solvent-matrix tube shaped around the Th molecule, favoring the upright arrangement of the molecules. DR-UV-vis spectra of the ZL/Th composites indicated that Th molecules are hosted in the ZL channels in a monomeric form. Photoluminescence spectroscopy demonstrated that photoluminescent dyes correspond to ca. $5 \%$ of the total amount of Th molecules in the zeolite porosities. While most of the photoluminescent Th molecules exhibit a lifetime equivalent to Th in solutions, the complementary fraction of photoluminescent Th exhibit significantly longer lifetimes, resulting from an entrapment in defects of the zeolite structure, inducing strong rotational constraints to the molecules.
\end{abstract}

\section{INTRODUCTION}

The physicochemical process of light conversion is under extensive investigation in order to develop more efficient devices for artificial photosynthesis, ${ }^{1-3}$ water splitting, photovoltaic applications, ${ }^{4}$ and targeting therapeutic agents. ${ }^{5-7}$ The photoprocesses of these systems are induced by nanostructured functional materials, such as zeolites light sensitized by dye molecules adsorbed into their pores allowing for the supramolecular organization of the photoactive species. Neutral and cationic dye molecules were successfully incorporated into various zeolites with suitable channel dimensions, such as $\mathrm{AlPO}_{4}-5,{ }^{8,9}$ zeolite $\mathrm{Y},{ }^{10}$ and zeolite $\mathrm{L}^{11,12}$ as well as mesoporous materials like MCM-41. ${ }^{13}$ Zeolite L (ZL) presents itself as an ideal host matrix because its arrays of parallel channels impose severe space restrictions and geometrical constraints to any inserted guest species, leading to very high concentrations of well-oriented dye molecules. Although dye-zeolite systems have been extensively reported in the literature ${ }^{5-7,14-17}$ and investigated experimentally-mainly by UV/vis spectroscopy, fluorescence microscopy, and IR/Raman spectroscopy ${ }^{18-21}-$ as well as theoretically, ${ }^{22-24}$ the influence of the zeolite pore systems on the dye arrangement and the potential molecules aggregation has not been yet fully investigated from a structural point of view by X-ray powder diffraction (XRPD).

Many factors influence the supramolecular organization of guest molecules, such as their size, shape, charge, and concentration, as well as the $\mathrm{Si} / \mathrm{Al}$ ratio of the host framework and the nature/amount of the extra-framework species. To date, only a few X-ray powder and single crystal diffraction studies endeavored a detailed structural characterization ${ }^{25-28}$ of dye-zeolite host-guest systems. Hennessy et al. ${ }^{25}$ characterized the arrangement of the cationic dye methyl viologen 
$\left(\mathrm{MV}^{2+}\right)$ in zeolite L. They combined X-ray powder diffraction, IR, and Raman spectroscopy and molecular modeling to localize the molecule in the channels, determined the amount of loaded dye, and proposed a slightly inclined orientation of the molecule with respect to the channel axis. Gigli et al. ${ }^{29}$ used XRPD, IR spectroscopy, and molecular modeling ${ }^{22-24}$ to determine the structure of zeolite L (ZL hereafter) loaded with high amounts of the neutral dye fluorenone. The theoretical and experimental results revealed a very densely packed dye arrangement, confirming the stability of the composite derived by the strong interaction between the dyes $\mathrm{C}=\mathrm{O}$ group and the $\mathrm{K}^{+}$ions present in the channels. ${ }^{24,29}$

The family of phenothiazine derivatives (thionine $=\mathrm{Th}$ hereafter, methylene blue $=\mathrm{MB}$ hereafter, azure $\mathrm{A}$ and azure $\mathrm{B}$ ) attracted considerable attention over the years due to their appropriate biological, chemical, photochemical, and photophysical properties ${ }^{30}$ which led them to be used as a model for phototherapeutic agents in photodynamic therapy (PDT) as well as for dye-sensitized solar energy converter. ${ }^{31,32}$ Calzaferri et al. ${ }^{33}$ performed first kinetics experiments on the incorporation of $\mathrm{Th}, \mathrm{MB}$, and ethylene blue into $\mathrm{ZL}$. They demonstrated the successful incorporation of $\mathrm{Th}$ into the zeolite channels, estimating that the dye can exchange at most $7.5 \%$ of the $\mathrm{K}^{+}$cations sited in the channel. Later studies on the aggregation properties of $\mathrm{Th}$ molecules within zeolites indicated that the channels cannot accommodate Th dimers due to space-filling effect and that the composite showed intense fluorescence. ${ }^{34}$ The same authors reported that thionine forms aggregates with the coguest dye molecule phenanthrene in the cavities of zeolite $\mathrm{Y}^{34}$

The nature of the aggregation of thionine and other related dyes (methylene blue, oxazine 170, nile blue A, acridine orange, pyronin-Y, and cresyl violet) within zeolites is controlled by different factors, chief among them the presence of water. Karuppannan et al. ${ }^{35}$ studied the different optical behavior of Th and MB encapsulated into zeolites Y, ZSM-5, and MCM-41 in absence and presence of $\mathrm{TiO}_{2}$ nanoparticles by applying absorption as well as picosecond and femtosecond timeresolved fluorescence techniques. They stated that the size, acidity, and hydrophilicity of the nanochannels and nanocavities of the specific zeolite influence the aggregation and the emission lifetime of Th. For example, Th encapsulated in $\mathrm{TiO}_{2}$ loaded ZSM-5, in the absence of water, is present in two excited states species undergoing excited state interconversion, while Th encapsulated in the large pore of the MCM-41 does not show this behavior, probably due to the presence of water molecules.

Knowledge of the geometrical arrangement of dye molecules and their chemical environment in the zeolite pores is a fundamental tool to understand the functionality of the hostguest systems. It also helps in elucidating their thermodynamic and kinetic stability, which is central to increasing the efficiency of the systems for energy transfer processes.

Simoncic et al. ${ }^{26,36}$ reported two single crystal X-ray studies on $\mathrm{Th}$ and $\mathrm{MB}$ incorporated into mordenite-Na. A slightly tilted arrangement of the Th molecules in the channels favors strong host-guest interactions between $\mathrm{C}, \mathrm{N}$, and $\mathrm{S}$ atoms of the dyes with the framework oxygen atoms. On the other hand, MB molecules were located in two partially occupied sites: one oriented nearly upright and the other slight inclined within the 12-member channel. Molecular mechanics studies on the orientation and dynamic diffusion of $\mathrm{Th}$ incorporated in mordenite were also performed by Deore et al. ${ }^{37}$ The authors report that Th molecules during the diffusion along the main channel of mordenite assume a centered and upright position, even if one does not consider water molecules. Once the diffusion is completed, the dye molecules bind to the channel wall in the orientation reported by Armbruster et al. ${ }^{26}$

In this study, we report a detailed structural characterization of the organization of the cationic dye Th inserted into ZL channels, which is carried out by XRPD and augmented with FT-IR spectroscopy for the determination of the host-guest interactions as well as by absorption UV-vis and photoluminescence spectroscopies for the investigation of the electronic states of the guest.

\section{EXPERIMENTAL SECTION}

2.1. Materials. The potassium $\mathrm{ZL}\left(\mathrm{K}_{8.46}\left(\mathrm{Al}_{8.35} \mathrm{Si}_{27.53}\right) \mathrm{O}_{72}\right.$. $17.91 \mathrm{H}_{2} \mathrm{O}$-LTL-framework type, ${ }^{38} \mathrm{Si} / \mathrm{Al}$ ratio 2.9 , space group P6/mmm, $a=18.3795(4), c=7.5281(2), V=$ 2202.4(1) (Table 1)-used for this study was purchased

Table 1. Experimental and Refinement Parameters for ZL As-Synthesized as Well as $\mathrm{ZL} / 0.15 \mathrm{Th}$ and $\mathrm{ZL} / 0.27 \mathrm{Th}$ Composites

\begin{tabular}{llll}
\multicolumn{1}{c}{ samples } & $\mathrm{ZL}($ ref 29$)$ & $\mathrm{ZL} / 0.15 \mathrm{Th}$ & $\mathrm{ZL} / 0.27 \mathrm{Th}$ \\
\hline space group & $P 6 / \mathrm{mmm}$ & $P 6 / \mathrm{mmm}$ & $P 6 / \mathrm{mmm}$ \\
$a(\AA)$ & $18.3795(4)$ & $18.3816(3)$ & $18.3866(2)$ \\
$c(\AA)$ & $7.5281(2)$ & $7.5283(2)$ & $7.5281(2)$ \\
$V\left(\AA^{3}\right)$ & $2202.4(1)$ & $2202.9(1)$ & $2204.0(1)$ \\
$R_{\mathrm{p}}(\%)$ & 2.5 & 2.6 & 2.7 \\
$R_{\mathrm{wp}}(\%)$ & 3.6 & 3.6 & 3.7 \\
$R F^{* * 2(\%)}$ & 6.6 & 6.5 & 7.6 \\
no. of variables & 64 & 89 & 89 \\
no. of observations & 2211 & 2225 & 2223 \\
no. of reflections & 936 & 955 & 955 \\
\hline
\end{tabular}

from Tosoh Corporation (Japan). The chemical formula was obtained by X-ray fluorescence (XRF). Zeolite L presents large circular 12-ring (12MR) channels of size $7.4 \times 7.8 \AA^{39-41}$ and smaller elliptical 8-ring (8MR) channels of size $1.9 \times 5.6 \AA$, both running along the $c$-axis (Figure 1). The main channels are connected to the parallel $8 \mathrm{MR}$ ones by nonplanar boat-shaped 8-membered rings.

Thionine acetate salt $\left(\mathrm{C}_{12} \mathrm{H}_{10} \mathrm{~N}_{3} \mathrm{~S}^{+}, 90 \%\right)$, was purchased from Sigma-Aldrich. Thionine, a metachromatic dye, is a flat molecule bearing a positive charge on the sulfur atom. Its dimensions, considering van der Waals radii, are $7.2 \times 15 \AA$, and its structure is reported in Figure 2.

2.2. Samples Preparation. Thionine incorporation was carried out by ion exchange, following the procedure described in refs 26 and 33. In a typical experiment, $60 \mathrm{mg}$ of ZL powder was dispersed in either 6.3 or $15.7 \mathrm{~mL}$ of a $3.3 \times 10^{-4} \mathrm{M}$ aqueous thionine acetate solution at $80^{\circ} \mathrm{C}$ for 3 days in a sealed ampule. After ion exchange, the blue powder was extensively washed: first three times with distilled water and then three times with ethanol to remove molecules adsorbed on the $\mathrm{ZL}$ surface. In this way two composites with different Th loading (labeled ZL/0.15Th and ZL/0.27Th) were obtained. Chemical, structural, and physical properties of the samples were determined by using the methods listed in the following section. 
a)

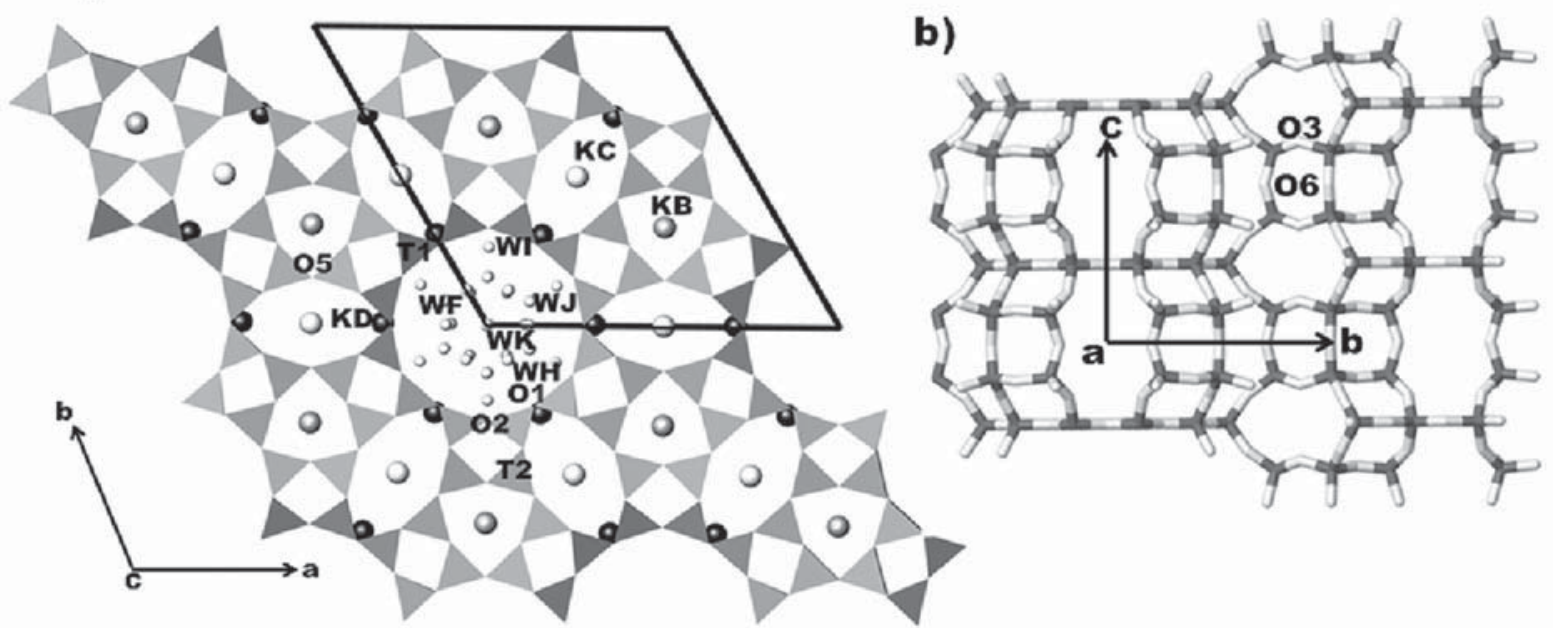

Figure 1. Projection of as-synthesized zeolite L structure and its unit cell along [001] (a) and along [110] (b). Light gray: water sites; dark gray: K sites.

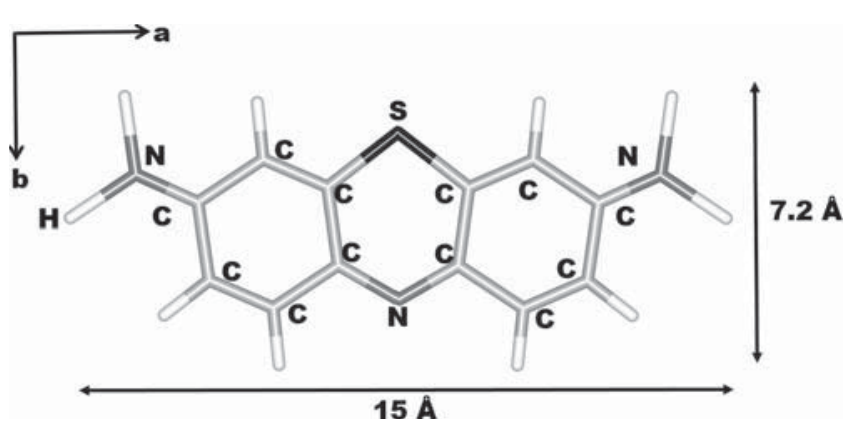

Figure 2. Structure and dimensions of thionine molecule.

\section{METHODS}

3.1. Thermogravimetric Analyses and Evolved Gas Mass Spectrometry (TGA-MSEGA). TGA-MSEGA analysis of as-synthesized $\mathrm{ZL}$, pure Th, and the two $\mathrm{ZL} / \mathrm{Th}$ composites were performed on a Seiko SSC 520 thermal analyzer equipped with a quadrupole mass spectrometer (ESS, GeneSys Quadstar 422) using the following experimental conditions: heating rate $10^{\circ} \mathrm{C} / \mathrm{min}$, temperature range $\mathrm{RT}-900{ }^{\circ} \mathrm{C}$, air flux of $100 \mu \mathrm{L} /$ min. The gas emitted during the thermal reactions was monitored in order to allow for the unambiguous identification of the species responsible of the weight loss observed in the TGA curve. Gas analyses were carried out in multiple ion detection (MID) mode, following the intensity changes of eight species $\left(m / z=18\left(\mathrm{H}_{2} \mathrm{O}\right), 44\right.$ and $45\left(\mathrm{CO}_{2}\right), 45.98\left(\mathrm{NO}_{2}\right), 52$ $\left(\mathrm{C}_{4} \mathrm{H}_{4}\right), 64\left(\mathrm{SO}_{2}\right), 76\left(\mathrm{~N}_{2} \mathrm{O}_{3}\right)$, and $\left.78\left(\mathrm{C}_{6} \mathrm{H}_{6}\right)\right)$ vs temperature. Before starting $\mathrm{MID}$ analysis, background subtraction was applied to set the zero point conditions.

3.2. IR-ATR Spectroscopy. Fourier transformed infrared spectra in air $\left(2 \mathrm{~cm}^{-1}\right.$ resolution, average on $\left.256 \mathrm{scans}\right)$ were collected in attenuated total reflection (ATR) mode on loose powder pressed on the internal reflection element (diamond) of a Bruker OPTIK platinum ATR accessory placed in a Bruker Vertex70 instrument (DTGS detector). The relative intensity of the bands over the spectral region considered was corrected for the different decay in the sample of the evanescent wave as a function of the incident wavelength, by dividing the original band intensities by the corresponding absorption wavelength. Atmospheric carbon dioxide and moisture signals have been subtracted from all the spectra by applying the Atmospheric Correction tools, as implemented in the Opus 6.5 software.

3.3. XRPD. XRPD patterns of the as-synthesized ZL, ZL/Th composites, and the pure dye were collected at the SNBL (BM01a) beamline of ESRF (European Synchrotron Radiation Facility) in transmission geometry, with a fixed wavelength of $0.6825 \AA$. The powder samples were loaded and packed in a 0.3 $\mathrm{mm}$ boron capillary, mounted on a standard goniometric head, and spun during data collection. The bidimensional diffraction patterns were recorded on a PILATUS3M Series detector (pixel dimension $172 \mu \mathrm{m}$ ) at a fixed distance of $193 \mathrm{~mm}$ from the sample. One-dimensional diffraction patterns were obtained in the $2 \theta$ range $0-40^{\circ}$ by integrating the two-dimensional images with the program FIT2D. ${ }^{42}$ Structural refinementswere performed by full profile Rietveld analysis using the GSAS package $^{43}$ with EXPGUI interface. ${ }^{44}$ Notwithstanding the length of Th molecule (15 $\AA$ ) - almost corresponding to the double of the ZL uc length along the $c$-axis $(7.5 \AA)$ - the diffraction data did not reveal the presence of superstructure and symmetry change in the ZL/Th composites. The refinements were performed in the parental ZL space group $P 6 / \mathrm{mmm}$. The framework and potassium atom coordinates reported in ref 41 for the room temperature refinement were used as starting model, and the extraframework atoms were derived by inspection of the Fourier difference maps. The Bragg peak profile was modeled using a pseudo-Voigt function with $0.01 \%$ cutoff peak intensity. The background curve was fitted using a Chebyschev polynomial with 23 variable coefficients. The 2 $\theta$-zero shift was accurately refined into the data set pattern. The scale factor and unit-cell parameters were allowed to vary for all cycles. The refined structural parameters for each data histogram were the following: same thermal displacement value for all tetrahedral atoms, a second value for all framework oxygen atoms, and a third one for the Th molecule atoms. The thermal parameters of $\mathrm{K}$ sites and oxygen atoms of water molecules were allowed to vary. Occupancy factors and isotropic thermal displacement factors were refined in alternate refinement cycles. Soft constraints were imposed on $\mathrm{Si}-\mathrm{O}$ (1.63 $\AA), \mathrm{C}-\mathrm{C}(1.39-1.48 \AA), \mathrm{S}-\mathrm{C}(1.63-1.74 \AA)$ and $\mathrm{C}-\mathrm{N}$ (1.26-1.33 $\AA$ ) distances during the first stages of the refinements and set free in the last cycles with tolerance values 
of $0.03 \AA$. The occupancy factors of Th sites were allowed to vary in the first refinement cycles and were successively constrained to an average value.

The final atomic positions and thermal parameters for the two composites (ZL/0.15Th and $\mathrm{ZL} / 0.27 \mathrm{Th}$ ) are given in Table S1, while the interatomic distances are reported in Table S2.

3.4. UV-Vis Absorption Spectroscopy. UV-vis-NIR measurements of the $\mathrm{ZL} / \mathrm{Th}$ samples were carried out in the diffuse reflection mode (DR) on a Varian Cary 5000 spectrometer equipped with an integrating sphere whose inner surface was coated with Spectralon. The spectra were acquired in air with loose powders diluted in pure ZL (1:50 mass ratio) placed inside the standard powder cell of the instrument. The absorption spectrum of thionine acetate was collected in transmission by using a $10^{-5} \mathrm{M}$ solution in ethanol in a Suprasil cuvette (optical path length: $10 \mathrm{~mm}$ ). Ethanol was chosen for this measurement, as it is the only solvent guaranteeing the solubilization of dye molecules in monomeric form at this concentration.

3.5. Photoluminescence Spectroscopy. Photoemission/ excitation steady-state spectra and fluorescence lifetimes of the $\mathrm{ZL} / \mathrm{Th}$ samples used above were recorded on a Horiba Jobin Yvon Fluorolog 3 TCSPC spectrofluorometer.

The samples were placed in a Suprasil quartz cuvette with an optical path length of $2 \mathrm{~mm}$. The spectrometer was equipped with a $450 \mathrm{~W}$ xenon lamp and a Hamamatsu R928 photomultiplier. A $10^{-7} \mathrm{M}$ solution of thionine acetate in ethanol was used to record the emission spectra of the thionine monomer in solution (in Suprasil cuvette, optical path length: $10 \mathrm{~mm}$ ). Fluorescence lifetimes were measured using a timecorrelated single photon counting (TCSPC) technique (Horiba Jobin Yvon), with a pulsed laser diode as excitation source at $560 \mathrm{~nm}$ (NanoLed, Horiba) and a pulse rate of $1 \mathrm{MHz}$ in a $90^{\circ}$ arrangement to a TBX-4 detector. The detector was set to 636 $\mathrm{nm}$, and the aperture of the band-pass was set to its maximum value $(24.9 \mathrm{~nm})$ due to the well-known weak fluorescence intensity of thionine samples. ${ }^{45,46}$ The instrument was set in the Reverse TAC mode, where the first detected photon represented the start signal for the time-to-amplitude converter (TAC), and the excitation pulse triggered the stop signal. DAS6 decay analysis software was used for lifetime calculation.

\section{RESULTS AND DISCUSSION}

4.1. TGA-MSEGA. TG and DTG spectra (Figure 3) clearly indicate intercalation of Th molecules into $\mathrm{ZL}$ channels. The amount of dye loaded in the two composites, as evaluated by the weight loss resulted in 0.15 and $0.27 \mathrm{Th}$ molecules per uc (Table 2). Calzaferri et al. ${ }^{33}$ reported that thionine can replace at most about $7.5 \%$ of the potassium in the ZL main channel (KD site), which corresponds to 0.27 molecule per unit cell. This is consistent with our data and evidence that the ZL/ $0.27 \mathrm{Th}$ sample corresponds to the maximum possible dye loading (Table 2).

Figure 3 shows the TG (a) and DTG curves (b) as a function of temperature for the as-synthesized $\mathrm{ZL}$, pure crystalline $\mathrm{Th}$, and $\mathrm{ZL} / \mathrm{Th}$ composites. The thermal behavior of the assynthesized ZL-whose total weight loss is about $11.9 \%$ - was described in detail in ref 41 . In both composites the Th release occurs in one single, wide step in the temperature range of $320-590{ }^{\circ} \mathrm{C}$. The mass spectrometry results showed that $\mathrm{Th}$ is thermally decomposed to $\mathrm{CO}_{2}, \mathrm{NO}_{2}$, and $\mathrm{SO}_{2}$ species. The

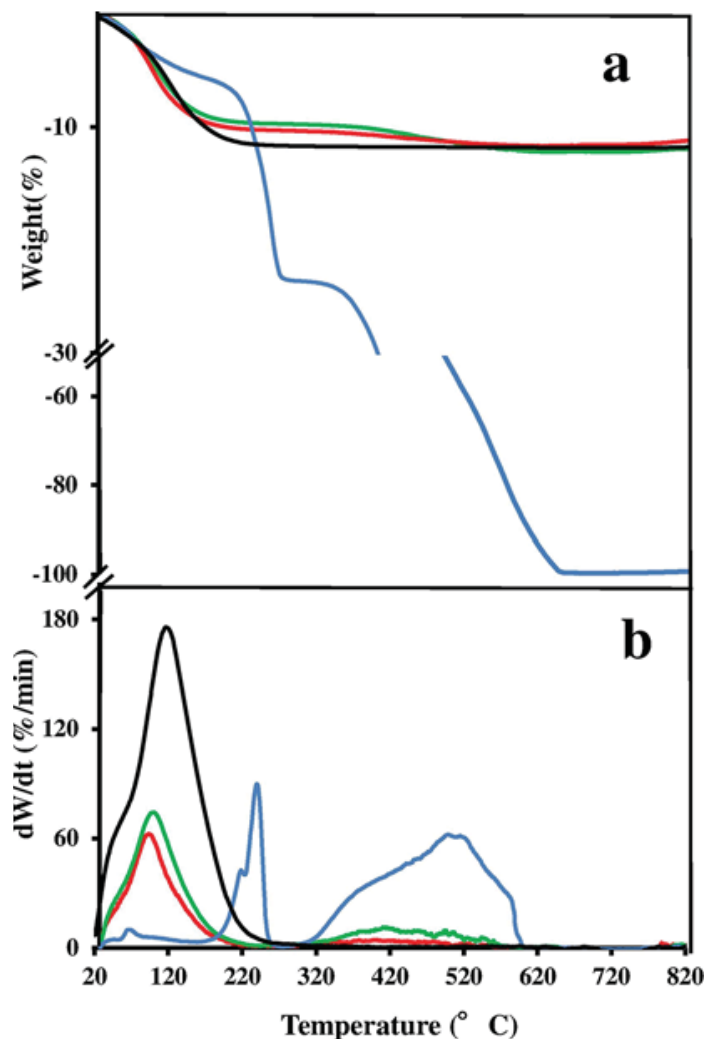

Figure 3. TG (a) and DTG curves (b) for the as-synthesized ZL (black solid curve), pure Th (blue curve), and the composites ZL/ $0.15 \mathrm{Th}$ (red line) and ZL/0.27Th (green line).

weight losses are resumed and interpreted for the two composites in Table 2.

The water weight losses (10\% and $9.5 \%$ observed in the composites $\mathrm{ZL} / 0.15 \mathrm{Th}$ and $\mathrm{ZL} / 0.27 \mathrm{Th}$, respectively, corresponding to 15.7 and 14.9 molecules per uc) are lower than that observed in ZL (18 molecules per uc) (Figure 3 and Table 2). This can be attributed to the Th molecules taking up space in the $\mathrm{ZL}$ channels.

Nevertheless, the amount of water left and the amount of encapsulated Th are not exactly inversely correlated, indicating that thionine, entering the channels, removes only a limited amount of the original water.

4.2. IR-ATR Spectroscopy. Figure 4 shows the IR-ATR spectra of ZL/0.15Th (a, green) and ZL/0.27Th (b, blue) composites as well as of solid thionine acetate (c, black curve) and $\mathrm{ZL}$ (d, light gray curve) for comparison purposes. A summary of the assignment of the various signals is listed in Table S3. The spectra of the composites and bare zeolite were normalized with respect to the zeolite framework peak at 1001 $\mathrm{cm}^{-1}$; thus, differences in relative intensity among the signals due to water and dye molecules in the zeolite channels can be ascribed to actual differences in their amount and/or their decadic absorption coefficients. Basically, the spectra of the composites are close to a superposition of those of the bare $\mathrm{ZL}$ and of thionine ions, but some significant spectral features deserve a comment:

(i) Bands due to acetate anions (in particular, those in region of the spectra not occupied by zeolite or thionine cation modes such as $\nu \mathrm{CH}_{3}$ at ca. $2950 \mathrm{~cm}^{-1}$ and $\nu_{\text {asym }} \mathrm{COO}^{-}$at $1544 \mathrm{~cm}^{-1}$ ) are not present in the spectra of $\mathrm{ZL} / \mathrm{Th}$ composites, indicating 
Table 2. Temperatures of Water and Th Weight Loss as Well as Number of Molecules (Water and Th), Determined by TGAMSEGA, Compared with Those Obtained by the Rietveld Refinements for the ZL/Th Composites

\begin{tabular}{|c|c|c|c|c|c|c|c|c|}
\hline sample & $\begin{array}{l}\text { water loss } \\
T\left({ }^{\circ} \mathrm{C}\right)\end{array}$ & $\begin{array}{l}\text { water } \\
\text { (wt \%) }\end{array}$ & $\begin{array}{l}\text { water molecules } \\
\text { (puc) (TGA) }\end{array}$ & $\begin{array}{l}\text { water molecules (puc) } \\
\text { (structure refinement) }\end{array}$ & $\begin{array}{l}\text { Th loss } \\
T\left({ }^{\circ} \mathrm{C}\right)\end{array}$ & $\begin{array}{c}\text { Th } \\
\text { (wt \%) }\end{array}$ & $\begin{array}{l}\text { Th molecules } \\
\text { (puc) (TGA) }\end{array}$ & $\begin{array}{l}\text { Th molecules (puc) } \\
\text { (structure refinement) }\end{array}$ \\
\hline $\mathrm{ZL}^{29}$ & 110 & 11.9 & 18 & 18 & & & & \\
\hline ZL/0.15Th & 102 & 100 & 15.7 & 15.8 & $320-590$ & 1.5 & 0.15 & 0.19 \\
\hline ZL/0.27Th & 100 & 9.5 & 14.9 & 14.8 & $320-590$ & 2.7 & 0.27 & 0.30 \\
\hline
\end{tabular}

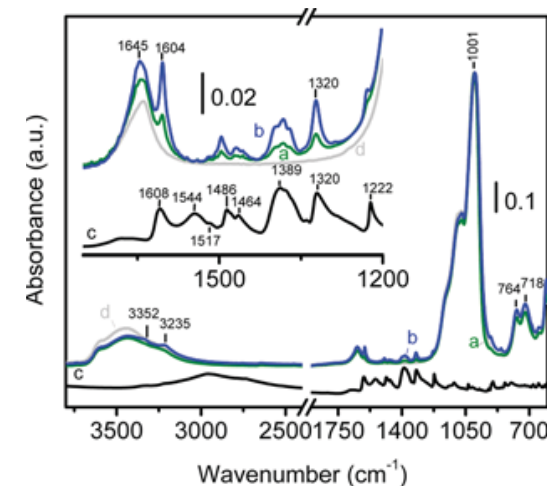

Figure 4. IR-ATR spectra of the ZL/0.15Th (a, green line) and ZL/ $0.27 \mathrm{Th}(\mathrm{b}$, blue line) samples. The inset shows a magnification of the $1750-1200 \mathrm{~cm}^{-1}$ region. The spectra for the solid thionine acetate (c, black line) and $\mathrm{ZL}$ (d, light gray line) in air are also reported for comparison.

that the negative zeolite framework is acting as counteranion for the encapsulated Th cations.

(ii) The peak at $1608 \mathrm{~cm}^{-1}$ in thionine acetate, due to a $\mathrm{C}-\mathrm{C}$ stretching mode of the aromatic rings, shifts to $1604 \mathrm{~cm}^{-1}$ in the composites and exhibits an increase in relative intensity with respect to the other dye modes. A similar behavior was previously reported for methyl blue after adsorption on titanates upon degassing and was considered associated with the direct interaction of $\pi$-orbitals with oxidic surfaces. ${ }^{47}$ On the other hand, the apparent reversing in the relative intensity of the bands at 1385 and $1320 \mathrm{~cm}^{-1}$ in the solid thionine acetate and in the $\mathrm{ZL} / \mathrm{Th}$ composites is due to the coexistence of signals related to thionine $(\nu \mathrm{C}-\mathrm{N}$ in the ring $)$ and to acetate $\left(\nu_{\mathrm{s}} \mathrm{COO}^{-}\right)$, the latter being no more important in the composites.

(iii) In the composite spectra, bands due to $\nu \mathrm{NH}_{2}$ stretching modes, located at 3352 and $3235 \mathrm{~cm}^{-1}$, exhibit a higher relative intensity. This should result from an increase in the polarization of the $\mathrm{N}-\mathrm{H}$ bonds, suggesting the $-\mathrm{NH}_{2}$ groups act as hydrogen-bond donors.

(iv) The broad signal due to the $\nu \mathrm{OH}$ of water molecules in the composites, spread over the $3600-3000 \mathrm{~cm}^{-1}$ range, appears similar in intensity for the two materials (slightly lower in the case of $\mathrm{ZL} / 0.15 \mathrm{Th}$ ) but weaker than for the bare $\mathrm{ZL}$, in agreement with the lower content in $\mathrm{H}_{2} \mathrm{O}$ molecules as indicated by TGA measurements. The opposite occurs for the $\delta \mathrm{H}_{2} \mathrm{O}$ signal: the main difference occurs again between bare $\mathrm{ZL}$ and $\mathrm{ZL} / 0.15 \mathrm{Th}$, with respect to this latter and $\mathrm{ZL} / 0.27 \mathrm{Th}$. The band is also slightly shifted to higher frequency ( 1645 $\mathrm{cm}^{-1}$ ). The rationalization of this effect is not straightforward, but it seems reasonable to associate it to an interaction among water and dye molecules hosted in the zeolite channels (see Figure 4).

4.3. Structure Refinement. The comparison between the XRPD patterns collected from ZL (see ref 29) and from the $\mathrm{ZL} / \mathrm{Th}$ composites (Figure S1) shows slight differences in the intensity of some diffraction peaks, particularly in the low $2 \theta$ angle region, which is the most sensitive to the extraframework content and distribution. These differences are consistent with the Th penetration and in agreement with the TGA and ATRIR spectroscopy results. The unit cell dimensions of both composites show an expansion along the $a$-axis with respect to the unloaded ZL (Table 1). This can be attributed to the penetration of the Th molecules, inducing a decrease of the ellipticity of the 8-membered rings perpendicular to [110] (Table 3). A small decrease of the ditrigonality of the D6R after the adsorption of $\mathrm{Th}$ is also observed (Table 3 ).

The structural refinements of the ZL/0.15Th and ZL/ 0.27Th composites gave similar results and are hence described here together. The configuration of zeolite L-with its high 6fold symmetry-and the low amount of dyes consisting of light atoms made molecule localization difficult. However, although the Fourier differences for both composites map showed many residual electronic density peaks, it was possible to define the whole geometry of the molecule and localize it in the channel. In both composite materials, the Th molecule is aligned parallel to the 12MR channel axis and is sited on the mirror planes parallel to the $c$-axis. In Figure 5 only one of the six possible orientations of the molecule and the organization of the water molecules are represented for the two samples. An amount of 0.19 and $0.30 \mathrm{Th}$ molecules per uc trapped in the zeolite porosities were estimated from the structural analysis for the $\mathrm{ZL} / 0.15 \mathrm{Th}$ and $\mathrm{ZL} / 0.27 \mathrm{Th}$ composites, respectively. The variation in the occupancy factor of the $\mathrm{KD}$ site-the only one accessible to the Th molecule-decreases consistently with

Table 3. Ellipticity of the $12 \mathrm{MR}$ and $8 \mathrm{MR}$ Channels Parallel to [001] and of 8MR Channel Parallel to [110] and Shape of the 6Rings of the D6R before $(\mathrm{ZL})$ and after the Adsorption of Thionine (ZL/0.15Th and ZL/0.27Th Composites)

\begin{tabular}{|c|c|c|c|c|c|c|c|c|}
\hline & \multicolumn{3}{|c|}{ ellipticity } & & & \multirow{2}{*}{\multicolumn{2}{|c|}{ D6R }} & \multirow[b]{2}{*}{$\mathrm{ID}^{a}$} \\
\hline & $12 \mathrm{MR}$ & $8 \mathrm{MR} \| \mathrm{c}$ & $8 \mathrm{MR} \perp[110]$ & & & & & \\
\hline & $(\mathrm{O} 2-\mathrm{O} 2 / \mathrm{O} 1-\mathrm{O} 1)$ & $(\mathrm{O} 1-\mathrm{O} 1 / \mathrm{O} 5-\mathrm{O} 5)$ & $(\mathrm{O} 1-01 / 06-06)$ & $\mathrm{O} 3-\mathrm{O} 3$ & $\mathrm{O} 5-\mathrm{O} 5$ & $\mathrm{O} 3-\mathrm{O} 5-\mathrm{O} 3$ & $\mathrm{O} 5-\mathrm{O} 3-\mathrm{O} 5$ & \\
\hline $\mathrm{ZL}$ & 1.03 & 1.70 & 1.61 & 3.84 & 4.97 & 94.83 & 144.64 & 1.29 \\
\hline ZL/0.15Th & 1.04 & 1.72 & 1.64 & 3.83 & 4.95 & 94.94 & 143.92 & 1.29 \\
\hline ZL/0.27Th & 1.04 & 1.67 & 1.32 & 3.81 & 4.83 & 96.55 & 142.17 & 1.27 \\
\hline
\end{tabular}

${ }^{a} \mathrm{Id}=$ ditrigonalization index of the $6 \mathrm{R}$ forming the $\mathrm{D} 6 \mathrm{R}$, given by the ratio between the longest and the shortest axis of the $6 \mathrm{R}$. 


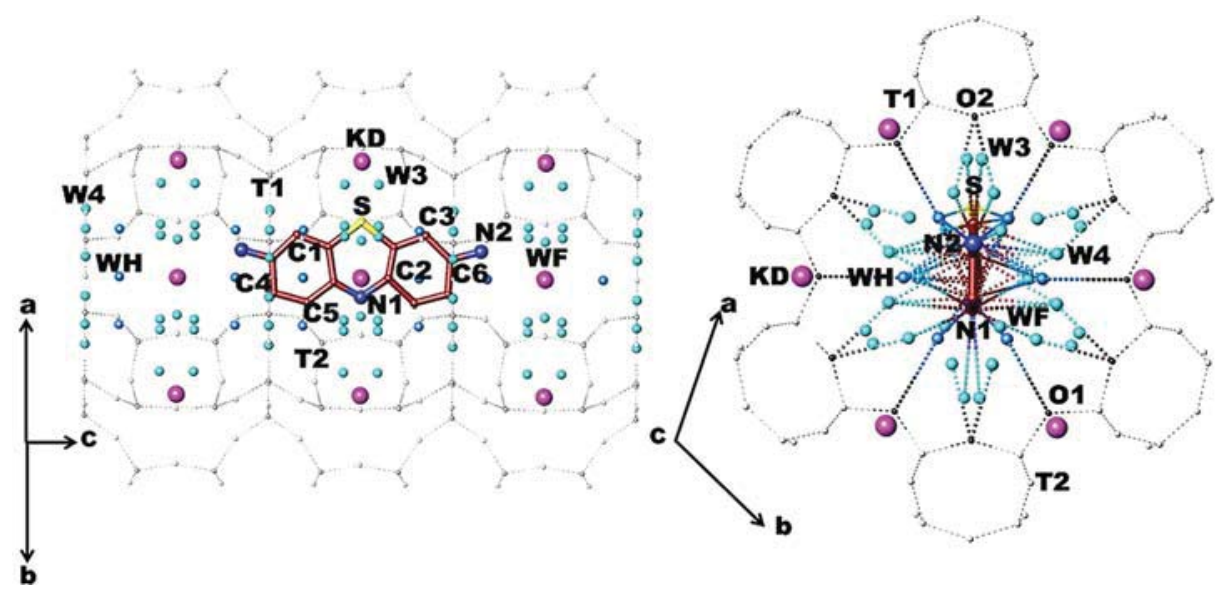

Figure 5. Arrangement of the thionine molecule and of the water-matrix surrounding the dye in the $\mathrm{ZL} / 0.15 \mathrm{Th}$ and $\mathrm{ZL} / 0.27 \mathrm{Th}$ composites viewed along [110] (a) and [001] (b), as obtained from structural refinements. Large fuxia spheres: $\mathrm{K}$ atoms; small light blue and turquoise spheres: water molecules (W). The distances between 2.5 and $3.2 \AA$ are drawn as dashed. The position of only one thionine molecule is represented for sake of clarity.

the Th-K exchange (Table S1), in agreement with that determined for the two composites by TGA (Table 2).

The water content of $\mathrm{ZL}$ corresponds to 18 molecules distributed over five extraframework sites located in the main 12MR channel, labeled WF, WH, WI, WJ, and WK (Figure 1), as reported by Gigli et al. ${ }^{41}$ These sites are partially occupied and weakly bound to the framework. The distribution of the residual water molecules in the two composites differs significantly from that of the unloaded ZL. In these composites the water molecules are located in the $12 \mathrm{MR}$ channel in four partially occupied sites. Two of them, labeled $\mathrm{WH}$ and WF, correspond to those present in ZL, while other two, W3 and $\mathrm{W} 4$, are new. As a whole, 15.8 molecules/uc in $\mathrm{ZL} / 0.15 \mathrm{Th}$ and 14.8 molecules/uc in ZL/0.27Th composite were found, in good agreement with the TGA results (Table 3 ).

In the study of Simoncic et al. ${ }^{26}$ of the Th-mordenite-Na system, strong interactions were found between the $\mathrm{S}, \mathrm{N}$, and $\mathrm{C}$ atoms of the $\mathrm{Th}$ molecules and the mordenite framework oxygen atoms, favored by aslightly inclined arrangement of the dye molecule along the channel axis.

In our study, the structural data (Table S2) indicates short distances between the $\mathrm{C}, \mathrm{S}$, and $\mathrm{N}$ atoms and water molecule sites suggesting water-mediated Th-ZL interactions. ${ }^{53}$ In fact, N2-W4 (2.24-2.67, and 2.58-2.79 for ZL/0.15Th and ZL/ $0.27 \mathrm{Th}$, respectively) and $\mathrm{N} 2-\mathrm{WH}$ distances (3.01 and 3.05 for ZL/0.15Th and ZL/0.27Th, respectively) suggest that the $\mathrm{NH}_{2}$ groups act as hydrogen-bond donors, consistent with the results from the IR-ATR spectroscopy. Other strong interactions are found between $\mathrm{S}$ and $\mathrm{WH}$ (2.57 and 2.55 for ZL/0.15Th and $\mathrm{ZL} / 0.27 \mathrm{Th}$, respectively) and WF (2.95 and 2.93 for ZL/ $0.15 \mathrm{Th}$ and $\mathrm{ZL} / 0.27 \mathrm{Th}$, respectively) water molecules (see Table S1), in agreement with the changes in the vibrational features of $\mathrm{H}_{2} \mathrm{O}$ molecules in the composites.

Water molecules in the ZL pores interact strongly with the dye and form a kind of solvent-matrix tube shaped around the Th molecule (Figure 5), favoring an upright arrangement of the molecules inside the $12 \mathrm{MR}$ channels.

4.4. DR-UV-Vis Absorption Spectroscopy. DR-UVvis spectra of the $\mathrm{ZL} / \mathrm{Th}$ composites are reported in Figure 6. Both materials were diluted with pure ZL powder (1:50, mass ratio; see Experimental Section for details) in order to obtain signals, which, once converted by applying the Kubelka-Munk

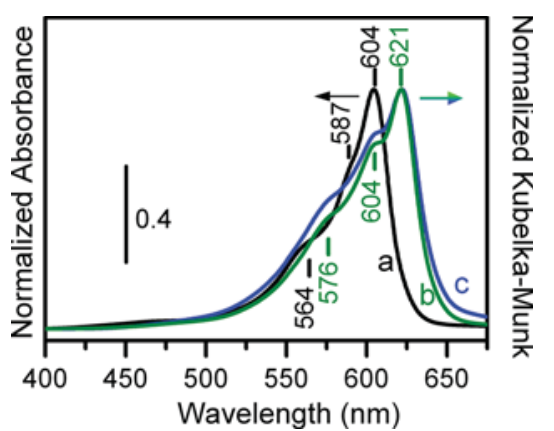

Figure 6. Electronic absorption spectra of (a) thionine acetate as $10^{-5}$ $\mathrm{M}$ ethanol solution (black curve), collected in the transmission mode; (b) $\mathrm{ZL} / 0.15 \mathrm{Th}$ (green curve); (c) $\mathrm{ZL} / 0.27 \mathrm{Th}$ (blue curve); the last two spectra were collected in the diffuse reflectance mode.

function, remained in the linear range of the relationship between intensity and concentration ( KM values $\leq 1$ ).

The spectrum of thionine acetate in ethanol solution (a, black curve) exhibits the typical profile expected for the monomeric form, with a main peak at $604 \mathrm{~nm}$ and two hypsochromic shoulders at ca. 587 and $564 \mathrm{~nm}$, due to $0 \leftarrow 0,1$ $\leftarrow 0$, and $2 \leftarrow 0 \pi^{*} \leftarrow \pi$ transitions. ${ }^{54}$ Looking at the ZL/ $0.15 \mathrm{Th}$ and $\mathrm{ZL} / 0.27 \mathrm{Th}$ composites (b, green curve, and c, blue curve, respectively), the absorption spectra appear almost coincident each other in position and shape, indicating $\mathrm{Th}$ molecules basically experience the same environment independently of the loading, which actually is rather low in both cases. Interestingly, the spectral profile of both composites exhibits a similar shape with respect to Th molecules in solution but appeared red-shifted by $17 \mathrm{~nm}$, indicating that the entrapment in the host affected the electronic states of the dye guest. This similarity in shape and the absence of a specific absorption peak at ca. $550 \mathrm{~nm}$ typical for thionine dimers $(\mathrm{H}$ aggregates)-found in the spectra of Th composites with zeolite with larger pores and higher cation content (ref 35)indicates that in this case the Th molecules are present in a monomeric form in the $\mathrm{ZL}$ channels. Moreover, the better resolved vibronic structure in the $\mathrm{ZL} / \mathrm{Th}$ spectra indicates the occurrence of some freedom restriction of dye molecules hosted in the zeolite channels. The red-shift of absorption maxima, which is related to the decrease of the energy gap 
between ground and excited electronic states, might be due to a modification of molecular planarity. ${ }^{28}$ The deviation from planarity is difficult to evidence from by the XRPD structural refinement, since the electron density map shows an average situation and thus the Th molecule appears located on the mirror plane. As an alternative explanation, in the case of different hybrid systems containing cationic dyes, it has been observed that hydrogen bonds play a relevant role in the stabilization of both ground and excited electronic states. ${ }^{55}$ In this respect, XRD and FT-IR data showed a strong interaction among water and Th molecules hosted inside the channels; the redistribution of the charge in the electronic excited state might then increase the hydrogen bond donor character of $-\mathrm{NH}_{2}$ groups of Th molecules due to the interaction with water, stabilizing the LUMO and reducing the HOMO-LUMO energy gap.

4.5. Photoluminescence Spectroscopy. In the final part of the investigation, the photophysical behavior of $\mathrm{Th}$ molecules hosted in the zeolite channels was investigated. The steady-state photoluminescence spectra are displayed in Figure 7. When comparing the emission spectra of $\mathrm{Th}$ in

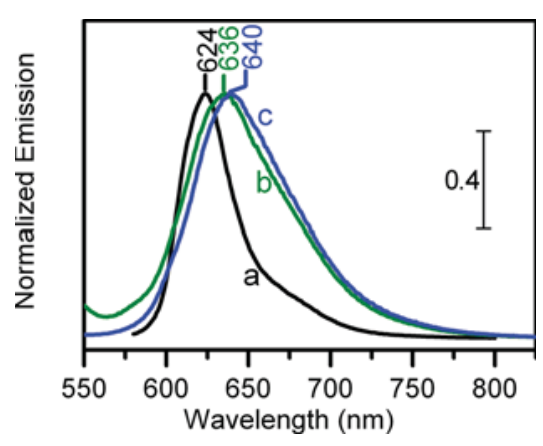

Figure 7. Normalized emission $\left(\lambda_{\text {exc }}=530 \mathrm{~nm}\right)$ spectra of (a) thionine acetate as a $10^{-7} \mathrm{M}$ ethanol solution (black curve), (b) $\mathrm{ZL} / 0.15 \mathrm{Th}$ (green curve), and (c) $\mathrm{ZL} / 0.27 \mathrm{Th}$ (blue curve); these two spectra were collected on samples diluted with bare ZL (1:50, mass ratio).

solution (a, black curve) to the $\mathrm{ZL} / 0.15 \mathrm{Th}$ and $\mathrm{ZL} / 0.27 \mathrm{Th}$ composites (b, green curve, and c, blue curve, respectively), a red-shift of the latter two emission maxima was observed, in agreement with the analogous shift of the absorption spectra. Moreover, the photoemission profiles of $\mathrm{Th}$ in composites appeared much broader on the longer wavelength side with respect $T h$ in solution, and this band broadening appears significantly larger than what was observed in the corresponding absorption spectra.

Photoluminescence spectra of both $\mathrm{ZL} / \mathrm{Th}$ composites were recorded on samples diluted in pure ZL until a 1:50 mass ratio, in order to avoid signals distortions due to interparticle selfabsorption effects; however, due to the high local molar thionine concentration $(<0.05 \mathrm{~mol} / \mathrm{L})$, the occurrence of intraparticle self-absorption cannot be excluded, ${ }^{14}$ likely explaining the slight red-shift observed by increasing Th loading. If the case, the occurrence of this phenomenon should prevent recording the true photoemission profile of photoluminescent Th molecules in the zeolite channels. Anyway, the photoluminescence spectra (or at least the part unaffected by self-absorption) of Th in composites appeared slightly broader than those the molecules in solutions, suggesting that the entrapment in the zeolite channel might have resulted in a modification of the shape of the potential well for the electronic ground state, with consequent changes in the probability of the radiative decays toward excited vibrational levels of this electronic state. Nevertheless, a modification only of the ground state is unlikely. However, changes in the shape of the potential well of the electronic excited state should have produced changes in the shape of the absorption spectra, and this was not the case. Hence, another possibility is that photoluminescent Th molecules in composites are only a small fraction of the total amount present in the zeolite channel, exhibiting a peculiar absorption profile included in the overall absorption spectrum. On this basis, the excitation spectra of Th in solution and of the composites were collected and compared with the correspondent absorption spectra (Figure 8, panels $\mathrm{A}$ and $\mathrm{B}$, respectively).

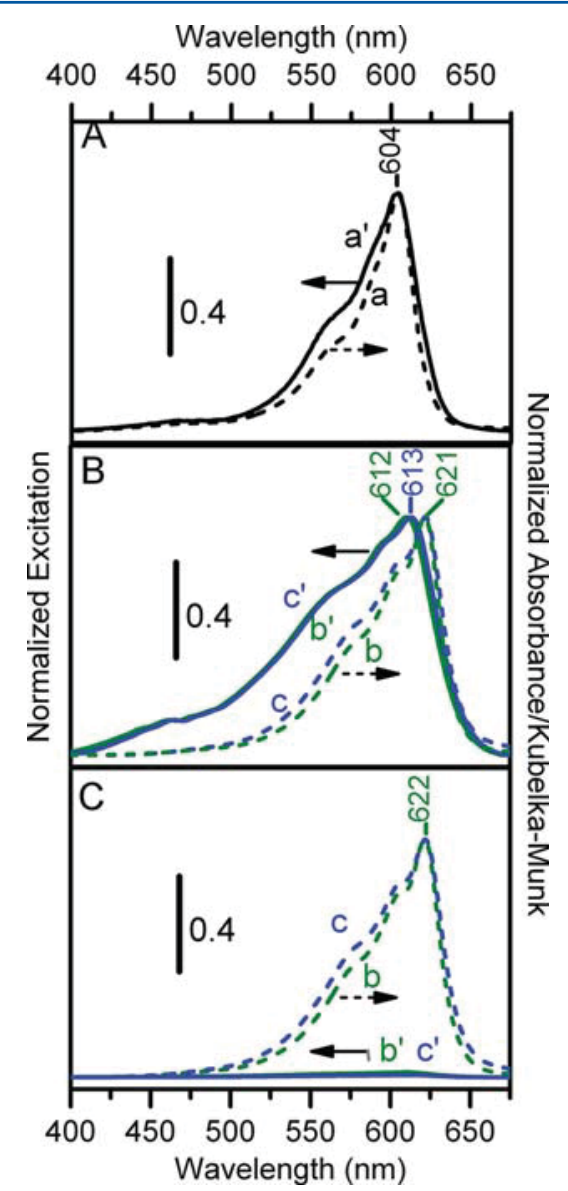

Figure 8. (A) Normalized absorption (a, dashed line) and excitation ( $\mathrm{a}^{\prime}$; solid line; $\lambda_{\mathrm{em}}=700 \mathrm{~nm}$ ) spectra of thionine acetate in an ethanol solution. (B) Normalized absorption (dashed lines) and excitation (solid lines, almost coincident for the two composites) spectra of the ZL/0.15Th ( $b$ and $b^{\prime}$, green curves) and ZL/0.27Th (c and $c^{\prime}$, blue curves) samples diluted in bare ZL (1:50 mass ratio). (C) Comparison of absorption (dashed lines) and excitation (solid lines) spectra of ZL/ $0.15 \mathrm{Th}$ ( $\mathrm{b}$ and $\mathrm{b}^{\prime}$, green curves) and ZL/0.27Th (c and $c^{\prime}$, blue curves). Excitation spectra were scaled to the same height as the absorption spectrum.

The absorption profiles of the photoluminescent molecules were acquired in this way. Minor differences were observed for Th in solution (panel A, curves a, $a^{\prime}$ ), whereas the excitation spectra of Th in the composites (panel B, curves $b^{\prime}, c^{\prime}$ ) appeared slightly blue-shifted and significantly broader toward shorter wavelengths than the corresponding absorption profiles 
(panel B, curves b, c), confirming that changes in the shape of the potential well for the electronic excited sate also occurred for Th photoluminescent molecules entrapped in the zeolite. It must now be considered that the absorption profile of these molecules, i.e., their excitation spectrum, should be included in the overall absorption profile of all Th molecules present in the zeolite channels. Thus, the intensities of the excitation spectra of ZL/0.15Th and ZL/0.27Th were properly scaled in order to be included under the corresponding absorption curves in the 400-680 nm range. The results are shown in Figure 8C: it appears that photoluminescent Th molecules in composites contribute in a very minor extent to the absorption spectra of these materials. More precisely, on the basis of the ratio among integrated areas, it can be proposed that actually photoluminescent dyes represent ca. $5 \%$ of the total amount of Th molecules in the zeolite channels. The fact that the overwhelming part of entrapped dye molecules appeared quenched can be the consequence of the interaction of their $\pi$-orbitals with the hosting environment, as proposed on the basis of the IR spectra (see above). Hence, the small fraction of photoluminescent molecules might correspond to dye molecules hosted in defect positions of the zeolite, where this interaction could be weaker. Moreover, time-resolved measurements (Table 4) indicated that more than $90 \%$ of photo-

Table 4. Photoemission Decay Times $\left(\tau^{\mathrm{F}}\right)$ of Thionine in Ethanol Solution $\left(10^{-7} \mathrm{M}\right)$ and of $\mathrm{ZL} / 0.15 \mathrm{Th}$ and $\mathrm{ZL} /$ 0.27Th Composites

\begin{tabular}{lcccc}
\multicolumn{1}{c}{ sample } & $\tau_{0}{ }^{\mathrm{F}}(\mathrm{ns})$ & $n_{1}(\%)$ & $\tau_{1}^{\mathrm{F}}(\mathrm{ns})$ & $n_{2}(\%)$ \\
thionine in solution & 0.65 & 100 & & \\
ZL/0.15Th & 0.65 & 93 & 1.85 & 7 \\
ZL/0.27Th & 0.68 & 97 & 1.95 & 3 \\
\hline
\end{tabular}

luminescent Th molecules in the composite exhibit a lifetime equivalent to Th molecules in solutions, suggesting they are weakly influenced by the host. Conversely, the complementary fraction of entrapped photoluminescent Th molecules exhibit significantly longer lifetimes, likely resulting from an entrapment in peculiar locations allowing severe constrains toward external rotational degree of freedom of the guest molecules.

The intrinsic limits of the diffraction methods-showing the average structure-combined with the low thionine loading hinder the localization of small fractions of molecules potentially located in structural defect sites.

\section{CONCLUSIONS}

It has been demonstrated in the literature that the encapsulation of dye molecules within a zeolite matrix can improve their optical properties such as photostability, luminescence quantum yields, and lifetimes. This study was driven by the need to understand whether the encapsulation of thionine with different loadings in ZL, along with its molecular arrangement, could affect its emitting properties.

The spectroscopic and structural results confirmed a maximum possible incorporation of thionine of 0.27 molecules-present as monomers-per unit cell.

Most of the Th molecules are weakly influenced by the host and exhibit a lifetime equivalent to Th molecules in solutions; on the other hand, a small fraction of molecules, entrapped in peculiar locations, which hinder the external rotational degree of freedom of the Th, show longer lifetimes.

\section{ASSOCIATED CONTENT}

\section{S Supporting Information}

Observed and calculated diffraction patterns and final difference curve from Rietveld refinements of the pure $\mathrm{ZL}$ and of the $\mathrm{ZL} /$ $0.15 \mathrm{Th}$ and $\mathrm{ZL} / 0.27 \mathrm{Th}$ composites; atomic coordinates, occupancy factors, and thermal displacement parameters for the ZL/0.15Th, ZL/0.27Th composites; extraframework bond distances for ZL/0.15Th and ZL/0.27Th, composites; the assignment of the various ATR-IR signals of pure compound Th and inside the ZL and bare ZL.

\section{AUTHOR INFORMATION}

\section{Corresponding Author}

*E-mail: Rossella.Arletti@unito.it (R.A.).

\section{Notes}

The authors declare no competing financial interest.

\section{ACKNOWLEDGMENTS}

The BM01 beamline at the European Synchrotron Radiation Facility and Dr. Vladimir Dmitriev are acknowledged for allocation of experimental beamtime. Dr. Simona Bigi is acknowledged for the help in TGA-MSEGA analyses. This work was supported by the Italian MIUR, within the framework of the project: FIRB, Futuro in Ricerca "Impose Pressure and Change Technology" (RBFR12CLQD); INFOCHEM project PRIN 2010CX2TLM 006.

\section{REFERENCES}

(1) Calzaferri, G. Nanochannels: Hosts for the Supramolecular Organization of Molecules and Complexes. Langmuir 2012, 28, 62166231

(2) Calzaferri, G.; Lutkouskaya, K. Mimicking the Antenna System of Green Plants. Photochem. Photobiol. Sci. 2008, 7, 879-910.

(3) Calzaferri, G.; Méallet-Renault, R.; Brühwiler, D.; Pansu, R.; Dolamic, I.; Dienel, T.; Adler, P.; Li, H.; Kunzmann, A. Designing Dye-Nanochannel Antenna Hybrid Materials for Light Harvesting, Transport and Trapping. ChemPhysChem 2011, 12, 580-594.

(4) Gust, D.; Moore, T. A.; Moore, A. L.Mimicking Photosynthetic Solar Energy Transduction. Acc. Chem. Res. 2001, 34, 40-48.

(5) Tsotsalas, M. M.; Kopka, K.; Luppi, G.; Wagner, S.; Law, M. P.; Schafers, M.; De Cola, L. Encapsulating (111)In in Nanocontainers for Scintigraphic Imaging: Synthesis, Characterization, and in Vivo Biodistribution. ACS Nano 2010, 4, 342-348.

(6) Li, Z.; Luppi, G.; Geiger, A.; Josel, H.-P.; De Cola, L. Bioconjugated Fluorescent Zeolite L Nanocrystals as Labels in Protein Microarrays. Small 2011, 7, 3193-3201.

(7) El-Gindi, J.; Benson, K.; De Cola, L.; Galla, H.-J.; Kehr, N. S. Cell Adhesion Behaviour on Enantiomerically Functionalized Zeolite L Monolayers. Angew. Chem., Int. Ed. 2012, 51, 3716-3720.

(8) Caro, J.; Marlow, F.; Wüst, M. Chromophore-Zeolite Composites: The Organizing Role of Molecular-Sieves. Adv. Mater. 1994, 6, 413-416.

(9) Ihlein, G.; Schuth, F.; Kraus, O.; Vietze, U.; Laeri, F. Alignment of a Laser Dye in the Channels of the $\mathrm{AlPO}_{4}-5$ Molecular Sieve. Adv. Mater. 1998, 10, 1117-1119.

(10) Hoppe, R.; Schulz-Ekloff, G.; Wöhrle, D.; Kirschhock, C.; Fuess, H.; Uytterhoeven, L.; Schoonheydt, R. Incorporation of Methylene Blue in NaY Zeolite at Crystallographically Defined Positions. Adv. Mater. 1995, 7, 61-64.

(11) Calzaferri, G.; Pauchard, M.; Maas, H.; Huber, S.; Khatyr, A.; Schaafsma, T. J. Photonic Antenna System for Light Harvesting, Transport and Trapping. J. Mater. Chem. 2002, 12, 1-13. 
(12) Mahato, R. N.; Lülf, H.; Siekman, M. H.; Kersten, S. P.; Bobbert, P. A.; de Jong, M. P.; De Cola, L.; van der Wiel, W. G. Ultrahigh Magnetoresistance at Room Temperature in Molecular Wires. Science 2013, 341, 257-260.

(13) Onida, B.; Bonelli, B.; Lucco-Borlera, M.; Flora, L.; Otero Arean, C.; Garrone, E. Spectroscopic Properties of Dye-Loaded Mesoporous Silicas of the Structural Type MCM-41. Stud. Surf. Sci. Catal. 2001, 135, 364-364.

(14) Devaux, A.; Calzaferri, G.; Miletto, I.; Cao, P.; Belser, P.; Brühwiler, D.; Khorev, O.; Häner, R.; Kunzmann, A. Self-Absorption and Luminescence Quantum Yields of Dye-Zeolite L Composites. J. Phys. Chem. C 2013, 117, 23034-23047.

(15) Calzaferri, G.; Huber, S.; Maas, H.; Minkowski, C. Host-Guest Antenna Materials. Angew. Chem., Int. Ed. 2003, 42, 3732-3758.

(16) Calzaferri, G.; Devaux, A. In Supramolecular Photochemistry; Ramamurthy, V., Inoue, Y., Eds.; Wiley \& Sons: New York, 2011.

(17) Fois, E.; Devaux, A.; Belser, P.; Brühwiler, D.; Calzaferri, G. Host-Guest Interactions and Orientation of Dyes in the OneDimensional Channels of Zeolite L. Langmuir 2013, 29, 9188-9198.

(18) Calzaferri, G. Organic-Inorganic Composites as Photonic Antenna. Chimia 2001, 55, 1009-1013.

(19) Maas, H.; Calzaferri, G. Constructing Dye-zeolite Photonic Nanodevices. Spectrum 2003, 16, 18-23.

(20) Devaux, A.; Minkowski, C.; Calzaferri, G. Electronic and Vibrational Properties of Fluorenone in the Channels of Zeolite L. Chem. - Eur. J. 2004, 10, 2391-2408.

(21) Megelski, S.; Lieb, A.; Pauchard, M.; Drechsler, A.; Glaus, S.; Debus, C.; Meixner, A. Orientation of Fluorescent Dyes in the Nano Channels of Zeolite L. J.; Calzaferri, G. J. Phys. Chem. B 2001, 105, $25-35$.

(22) Fois, E.; Tabacchi, G.; Devaux, A.; Belser, P.; Brühwiler, D.; Calzaferri, G. Host-Guest Interactions and Orientation of Dyes in the One-Dimensional Channels of Zeolite L. Langmuir 2013, 29, 91889198.

(23) Fois, E.; Tabacchi, G.; Calzaferri, G. Orientation and Order of Xanthene Dyes in the One-Dimensional Channels of Zeolite L: Bridging the Gap between Experimental Data and Molecular Behavior. J. Phys. Chem. C 2012, 116, 16784-16799.

(24) Fois, E.; Tabacchi, G.; Calzaferri, G. Interactions, Behavior and Stability of Fluorenone Inside Zeolite Nanochannels. J. Phys. Chem. C 2010, 114, 10572-10579.

(25) Hennessy, B.; Megelski, S.; Marcolli, C.; Shklover, V.; Bärlocher, Ch.; Calzaferri, G. Characterization of Methyl Viologen in the Channels of Zeolite L. J. Phys. Chem. B 1999, 103, 3340-3351.

(26) Simoncic, P.; Armbruster, T.; Pattison, P. Cationic Thionin Blue in the Channels of Zeolite Mordenite. J. Phys. Chem. B 2004, 108, $17352-17360$

(27) Van Koningsveld, H.; Tuinstra, F.; Van Bekkum, H.; Jansen, J. C. Single Crystal Structure Analysis of a High-loaded Complex of HZSM-5 with Para-dichlorobenzene. Acta Crystallogr., Sect. B: Struct. Sci. 1989, 45, 423-431.

(28) Ehrl, M.; Kindervater, H. W.; Deeg, F. W.; Braeuchle, C.; Hoppe, R. Optical Spectroscopy of Thiazine and Oxazine Dyes in the Cages of Hydrated and Dehydrated Faujasite-Type Zeolites: Molecular Dynamics in a Nanostructured Environment. J. Phys. Chem. 1994, 98, 11756-11763.

(29) Gigli, L.; Arletti, R.; Tabacchi, G.; Fois, E.; Vitillo, J. G.; Martra, G.; Agostini, G.; Quartieri, S.; Vezzalini, G. Close-Packed Dye Molecules in Zeolite Channels Self-Assemble into Supramolecular Nanoladders. J. Phys. Chem. C 2014, 118, 15732-15743.

(30) Moreira, L. M.; Lyon, J. P.; Romani, A. P.; Severino, D.; Rodrigues, M. R.; de Oliveira, H. P. M. Phenotiazinium Dyes as Photosensitizers (PS) in Photodynamic Therapy (PDT): Spectroscopic Properties and Photochemical Mechanisms; INTECH Open Access Publisher: 2012.

(31) Mellish, K. J.; Cox, R. D.; Vernon, D. I.; Griffiths, J.; Brown, S. B. In Vitro Photodynamic Activity of a Series of Methylene Blue Analogues. Photochem. Photobiol. 2002, 75, 392-7.
(32) Verbunt, P. P. C.; Debije, M. G. Progress in Luminescent Solar Concentrator Research: Solar Energy for the Built Environment; World Renewable Energy Congress: Sweden, 2011.

(33) Calzaferri, G.; Gfeller, N. Thionine in the Cages of Zeolite L. J. Phys. Chem. 1992, 96, 3428-3435.

(34) Ramamurthy, V.; Sanderson, D. R.; Eaton, D. F. Control of Dye Assembly within Zeolites: Role of Water. J. Am. Chem. Soc. 1993, 115, 10438-10439.

(35) Senthilkumar, K.; Paul, P.; Selvaraju, C.; Natarajan, P. Preparation, Characterization, and Photophysical Study of Thiazine Dyes within the Nanotubes and Nanocavities of Silicate Host: Influence of Titanium Dioxide Nanoparticle on the Protonation and Aggregation of Dyes. J. Phys. Chem. C 2010, 114, 7085-7094.

(36) Simoncic, P.; Armbruster, T. Cationic Methylene Blue Incorporated into Zeolite Mordenite-Na: a Single Crystal X-ray Study. Microporous Mesoporous Mater. 2005, 81, 87-95.

(37) Deore, S.; Simoncic, P.; Navrotsky, A. Molecular Mechanics Studies of Thionin Blue in Zeolite Mordenite. Microporous Mesoporous Mater. 2008, 109, 342-349.

(38) Baerlocher, Ch.; McCusker, L. B.; Olson, D. H. Atlas of Zeolite Framework Types, 6th ed.; Elsevier: Amsterdam, 2007.

(39) Barrer, R. M.; Villiger, H. Z. The Crystal Structure of the Synthetic Zeolite L. Z. Kristallogr. 1969, 128, 352-370.

(40) Newsam, J. M. Structures of Dehydrated Potassium Zeolite L at 298 and $78 \mathrm{~K}$ and at $78 \mathrm{~K}$ Containing Sorbed Perdeuteriobenzene. J. Phys. Chem. 1989, 93, 7689-7694.

(41) Gigli, L.; Arletti, R.; Quartieri, S.; Di Renzo, F.; Vezzalini, G. The High Thermal Stability of the Synthetic Zeolite KL: Dehydration Mechanism by in Situ SR-XRPD Experiments. Microporous Mesoporous Mater. 2013, 177, 8-16.

(42) Hammersley, A. P.; Svensson, S. O.; Hanfland, M.; Fitch, A. N.; Häusermann, D. Two Dimensional Detector Software: From Real Detector to Idealized Image or Two Theta Scan. High Pressure Res. 1996, 14, 235-248.

(43) Larson, A. C.; Von Dreele, R. B. General Structure Analysis System "GSAS"; Los Alamos National Laboratory Report; Los Alamos, 1994; LAUR 86-748.

(44) Toby, B.H. J. EXPGUI, a Graphical User Interface for GSAS. J. Appl. Crystallogr. 2001, 34, 210-213.

(45) Mackay, R. A.; Lai, W. C. Energy Transfer From Rhodamine. 6G to Thionine in a Anionic Microemulsion. Colloids Surf., A 2005, 254, 115-123.

(46) Rodriguez-Serrano, A.; Daza, M. C.; Doerr, M.; Marianc, C. M. A Quantum Chemical Investigation of the Electronic Structure of Thionine. Photochem. Photobiol. Sci. 2012, 11, 397-408.

(47) Horváth, E.; Ribic, P. R.; Hashemi, F.; Forróa, L.; Magrez, A. Dye Metachromasy on Titanate Nanowires: Sensing Humidity with Reversible Molecular Dimerization. J. Mater. Chem. 2012, 22, 87788784 .

(48) Colthup, N. B.; Daly, L. H.; Wiberley, S. E. Introduction to Infrared and Raman Spectroscopy, 2nd ed.; Academic Press: New York, 1975

(49) Nakamoto, K. Infrared and Raman Spectra of Inorganic and Coordination Compounds, 4th ed.;Wiley: New York, 1986.

(50) Hutchinson, K.; Heste, R. E. Raman Spectroscopic Studies of a Thionine-Modified Electrode. J. Chem. Soc., Faraday Trans. 1 1984, 80, 2053-2071.

(51) Bellamy, L. J. The Infra-red Spectra of Complex Molecules, 3rd ed.; Chapman and Hall: London, 1975.

(52) Jacobs, W. P. J. H.; van Wolput, J. M. C.; van Santen, R. A. An in Situ Fourier Transform Infrared Study of Zeolitic Vibrations: Dehydration, Deammoniation, and Reammoniation of Ion-Exchanged Y Zeolites. Zeolites 1993, 13, 170-182.

(53) Steiner, T. Water Molecules which Apparently Accept no

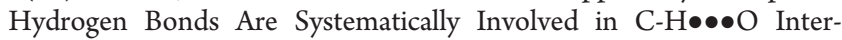
actions. Acta Crystallogr., Sect. D: Biol. Crystallogr. 1995, 51, 93-97.

(54) Lai, W. C.; Dixit, N. S.; Mackay, R. A. Formation of $\mathrm{H}$ Aggregates of Thionine Dye in Water. J. Phys. Chem. 1984, 88, 53645368 . 
(55) Epelde-Elezcano, N.; Duque-Redondo, E.; Martínez-Martínez, V.; Manzano, H.; López-Arbeloa, I. Preparation, Photophysical Characterization, and Modeling of LDS722/Laponite 2D-Ordered Hybrid Films. Langmuir 2014, 30, 10112-10117. 